
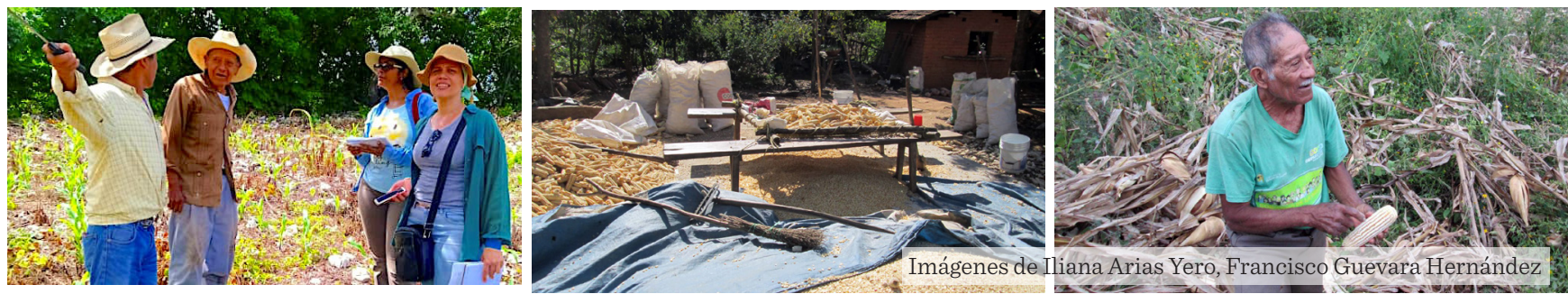

\title{
Caracterización y tipos de familias productoras de maíz local en la Frailesca, Chiapas
}

\section{Typological characterization of the maize producing families from la Frailesca, Chiapas}

Iliana Arias-Yero', Francisco Guevara-Hernández ${ }^{2 *}$, Manuel Alejandro La 0-Arias², Pedro Cadena-Iñiguez ${ }^{3}$

\section{RESUMEN}

Las Unidades de Producción Familiar (UPF) están conformadas por capitales natural, físico, financiero, humano y social. Los dos últimos constituyen la base del funcionamiento del resto y de la unidad de producción. En México, Chiapas y la Frailesca, la producción de maíces, particularmente de maíces locales, tiene valor cultural, agronómico y económico. El objetivo de esta investigación fue caracterizar las Unidades de Producción Familiar que cultivan maíces locales en la región Frailesca del estado de Chiapas, sobre la base cultural del manejo de los capitales humano y social. Se estudiaron $80 \mathrm{UPF}$ de 20 comunidades seleccionadas mediante un muestreo por redes. Se utilizó el enfoque Modos de Vida Sustentable como marco de referencia. Se aplicaron entrevistas semiestructuradas y se realizaron observaciones participantes. En el estudio predominaron las familias nucleares con líderes de edad avanzada. Los jóvenes no priorizan la conservación de los maíces locales sino actividades más rentables como la ganadería, los maíces híbridos y el café. Existe un excesivo uso de agroquímicos que afecta los recursos suelo, agua y biodiversidad. Se distinguieron 6 tipos de UPF de acuerdo con sus capitales humano y social. El grupo 3 (G3) mostró un mejor equilibrio entre ambos por la participación familiar, destacándose entre los de mayor experiencia en el cultivo de maíces locales y por tener relaciones locales y de cooperación más sólidas. Se concluye que las UPF presentan características que contribuyen o afectan la conservación de los maíces locales, como la continuidad generacional, el deterioro de los recursos naturales que sostienen el cultivo a través de la fusión de las prácticas tradicionales con las modernas y la dependencia de recursos externos.

PALABRAS CLAVE: capital humano, capital social, maíces locales, modos de vida, sustentabilidad.

\section{ABSTRACT}

The Family Production Units (FPU) are made up of natural, physical, financial, human and social capital. The last two constitute the basis for the functioning of the rest of the capitals and of the production unit. In Mexico, Chiapas and La Frailesca, the production of corn, and particularly local corn, has cultural, agronomic, and economic value. The objective of this research was to characterize the UPF that cultivate local corn, in the Frailesca region of the State of Chiapas, on the cultural basis of the management of human and social capital. 80 UPF from 20 communities were studied, selected through network sampling. The Sustainable Livelihoods approach was used as a frame of reference. Semi-structured interviews were applied, and participant observations were made. In the predominant study in nuclear families with elderly leaders predominated. Young people do not prioritize the conservation of local corn, but rather more profitable activities such as livestock, hybrid corn and coffee. There is an excessive use of agrochemicals that affects soil, water and biodiversity resources. Six types of UPF were distinguished according to their human and social capital. The third category (G3) shows a better balance between human and social capital due to their family involvement, standing out among those with more experience in growing local corn and having stronger local relationships. It is concluded that the UPF present characteristics that foster or impair the preservation of local corn such as generational continuity, the deterioration of the natural resources that sustain the cultivation through the fusion of traditional and modern practices and dependence on external resources.

KEYWORDS: human capital, social capital, local maize, livelihoods, sustainability.

*Correspondencia: francisco.guevara@unach.mx/ Fecha de recepción: 14 de enero de 2021/Fecha de aceptación: 17 de enero de 2022/ Fecha de publicación: 28 de enero de 2022.

${ }^{1}$ Universidad Autónoma de Chiapas, Doctorado en Ciencias Agropecuarias y Sustentabilidad (DOCAS), Tuxtla Gutiérrez, Chiapas, México. ${ }^{2}$ Universidad Autónoma de Chiapas, Facultad de Ciencias Agronómicas Campus V, carretera Ocozocoautla-Villaflores km 84.5, Apartado Postal 78, Villaflores, Chiapas, México, C.P. $30470 .{ }^{3}$ Instituto de Investigaciones Forestales Agrícolas y Pecuarias, Campo Experimental Centro de Chiapas, Ocozocoautla, Chiapas, México. 


\section{INTRODUCCIÓN}

Las Unidades de Producción Familiar (UPF) están asociadas a modos de vida de las familias rurales y a la agricultura familiar (Salcedo y Guzmán, 2014; García y col., 2015). En las últimas décadas han sido objeto de estudio en América Latina por las múltiples transformaciones que han enfrentado el medio rural y sus actores. En el presente trabajo se entendieron las UPF como una estructura socio-productiva básica, asociada a un modo de vida rural que parte de la interacción familia-parcela-entorno para garantizar la producción agrícola y la reproducción social de una familia. En esta interacción confluyen un conjunto de relaciones socioculturales y productivas y se generan estrategias con la articulación de sus recursos (capitales) que le permiten a la familia, a través del trabajo agropecuario y no agropecuario, su supervivencia (Maletta, 2011; van-der-Ploeg, 2013; FAO, 2018).

Las UPF representan pilares fundamentales para la alimentación y la economía en América Latina y el Caribe al producir aproximadamente el $80 \%$ del valor de los alimentos del mundo, de acuerdo con la Organización de las Naciones Unidas para la Alimentación y la Agricultura (FAO, por sus siglas en inglés: Food and Agriculture Organization). En esta región la agricultura familiar alcanza, a nivel de países, entre el $12 \%$ y el $67 \%$ de la superficie agropecuaria; 8 de cada 10 explotaciones en América Latina son de tipo familiar y el $35 \%$ de ellas se encuentran en América Central y México (FAO, 2018).

En México, el $40 \%$ de los granos básicos (maíz y frijol) provienen del trabajo de 5 millones de productores familiares, de acuerdo con el Servicio de Información Agroalimentaria y Pesquera (SIAP, 2020), para quienes el maíz (Zea mays L.) es el cultivo de mayor importancia y forma parte de su vida cotidiana. Por eso, una vez procesado, es el principal alimento para los mexicanos (Yúñez y col., 2013) y se caracteriza por su gran potencial de usos que le confiere un extraordinario valor cultural, sobre todo al maíz local, por sus características físicas, como el mejor tamaño de mazorca y peso de grano (Delgado y col., 2018), y culinarias, por ejemplo, la calidad del nixtamal y tortilla, sabor del elote y el pozol (Guevara y col., 2019; 2020).

Se entiende como maíces locales a los desarrollados a partir del conocimiento tradicional, ya sean nativos de una región específica o como resultado de la adopción, adaptación y conservación (tanto de la semilla como del cultivo) por más de dos años en un espacio geográfico determinado, con un valor e identidad cultural significativo para los productores y sus familias, que se trasmite generación tras generación (Guevara y col., 2019).

En la región Frailesca, Chiapas, México, con el Tratado de Libre Comercio de América del Norte (TLCAN), los medianos y pequeños productores de granos básicos fueron, de alguna forma, prácticamente excluidos del mercado, debido a que México comenzó la importación de productos subsidiados y con bajos aranceles desde Estados Unidos y precios inferiores a los nacionales (Rubio, 2012). Se observó así una disminución de la superficie sembrada de maíz, de 136405 ha en 2003 a 61365 en 2019, aunque con un incremento en el rendimiento de $2.85 \mathrm{~T} / \mathrm{ha}$ a $3.35 \mathrm{~T} / \mathrm{ha}$ (SIAP, 2020). Tal situación concuerda con el modelo de agricultura tecnificada promovida con la Revolución Verde desde la primera mitad del siglo XX, que propició, sobre todo en las regiones de producción de pequeña escala, la degradación de los suelos, la pérdida de prácticas culturales asociadas al cultivo y alta dependencia de insumos externos. Este panorama, a la vez, incidió en el deterioro de los ingresos y la calidad de vida de los agricultores y sus familias (Aguilar y col., 2019; Pizaña y col., 2019; Martínez y col., 2020b).

Particularmente, los maíces locales han sido los más afectados, por su rendimiento inferior a las generaciones avanzadas de híbridos introducidas por empresas semilleras como PIONEER y DEKALB, entre otras, desde hace más de 30 años (Guevara y col., 2019; 2020). Aun así, 
Guevara y col. (2020) identificaron 88 variedades de maíces locales conservados en la región. Esto denota la existencia de UPF que, a pesar de las campañas intensas y permanentes para posicionar las semillas mejoradas, conservan y mantienen el uso y producción de semillas locales, lo que evidencia la necesidad de un estudio que profundice en el conocimiento y las dinámicas de estas unidades de producción.

En ese tenor, existen precedentes sobre las investigaciones del agroecosistema relacionadas con actores, estrategias y dinámicas del agro (Cadena-Iñiguez y col., 2018a; 2018b; 2019), los usos múltiples del maíz (Hernández y col., 2017; Hernández y col., 2020), estudios etnográficos del sistema maíz y las UPF (Guevara y col., 2018a; 2020), actores y estrategias de la innovación tecnológica (Guevara y col., 2018b), caracterización de productores e indicadores de sustentabilidad del sistema maíz (Martínez y col., 2020c), entre otros. Dichos trabajos convergen en la relación dialéctica de los maíces locales con su entorno sociocultural y económico, lo que hace relevantes a los procesos coevolutivos que enlazan el proceso de adaptación local y el desarrollo de estos cultivos con las UPF que los sostienen.

Lo anterior fundamenta la importancia de profundizar en los estudios relacionados con la producción familiar y sus particularidades, sobre todo aquellas relacionadas con la toma de decisiones para el cultivo de los maíces locales. La caracterización de las UPF representa un punto de partida básico e importante para analizar sus lógicas y racionalidades, así como definir aquellos elementos clave para su fortalecimiento y desarrollo. Esto incluye el reconocimiento de su diversidad mediante la identificación de posibles tipos de UPF (Santos y col., 2014).

En ese sentido, el concepto de UPF trasciende a la visión de una unidad de producción, porque implica y relaciona la complejidad de un modo de vida, por lo que el marco conceptual Modos de Vida Sustentable (MVS) propuesto por el Servicio Jesuita a Refugiados (SJR, 2017), resulta propicio para conceptualizar las dimensiones de una posible caracterización. Desde esta perspectiva de análisis las UPF combinan tácitamente 5 capitales: humano, social, natural, financiero y físico; los dos primeros por definición son intangibles y están mediados culturalmente, los determina la dimensión cultural de las estrategias de supervivencia.

Los capitales humano y social resultan esenciales para articular el resto de los capitales (Aguilar y col., 2012; Gómez y col., 2013). El humano se refiere a los conocimientos locales y técnicos de los agricultores, las potencialidades, habilidades, experiencias, motivaciones, capacidades laborales, y algunos otros aspectos más, con los que cuentan tanto los seres humanos individualmente como los grupos. Por su parte, el capital social contempla el establecimiento de redes y conexiones, incluidas las instituciones, participación en grupos formales e informales, organizaciones y relaciones de confianza entre amigos, compadres y vecinos (SJR, 2017; Vázquez y col., 2018).

El objetivo de la presente investigación fue caracterizar las Unidades de Producción Familiar que cultivan maíces locales en la región Frailesca del estado de Chipas sobre la base cultural del manejo de los capitales humano y social.

\section{MATERIALES Y MÉTODOS}

\section{La región Frailesca}

La Frailesca está localizada entre la llanura costera del Pacífico y la depresión central de Chiapas. Se conforma por 6 municipios: Villa Corzo, Villaflores, La Concordia, Ángel Albino Corzo, El Parral y Montecristo de Guerrero (Figura 1), que en total ocupan una extensión territorial de $7987.19 \mathrm{~km}^{2}$. Existen 2949 localidades, de ellas, 2928 son rurales (Gobierno del estado de Chiapas, 2014).

La región es muy importante por su contribución a la economía estatal, ya que tiene como actividades principales la agricultura de temporal para maíz, con el $9 \%$ de la superficie sembrada, y el mayor rendimiento del esta- 

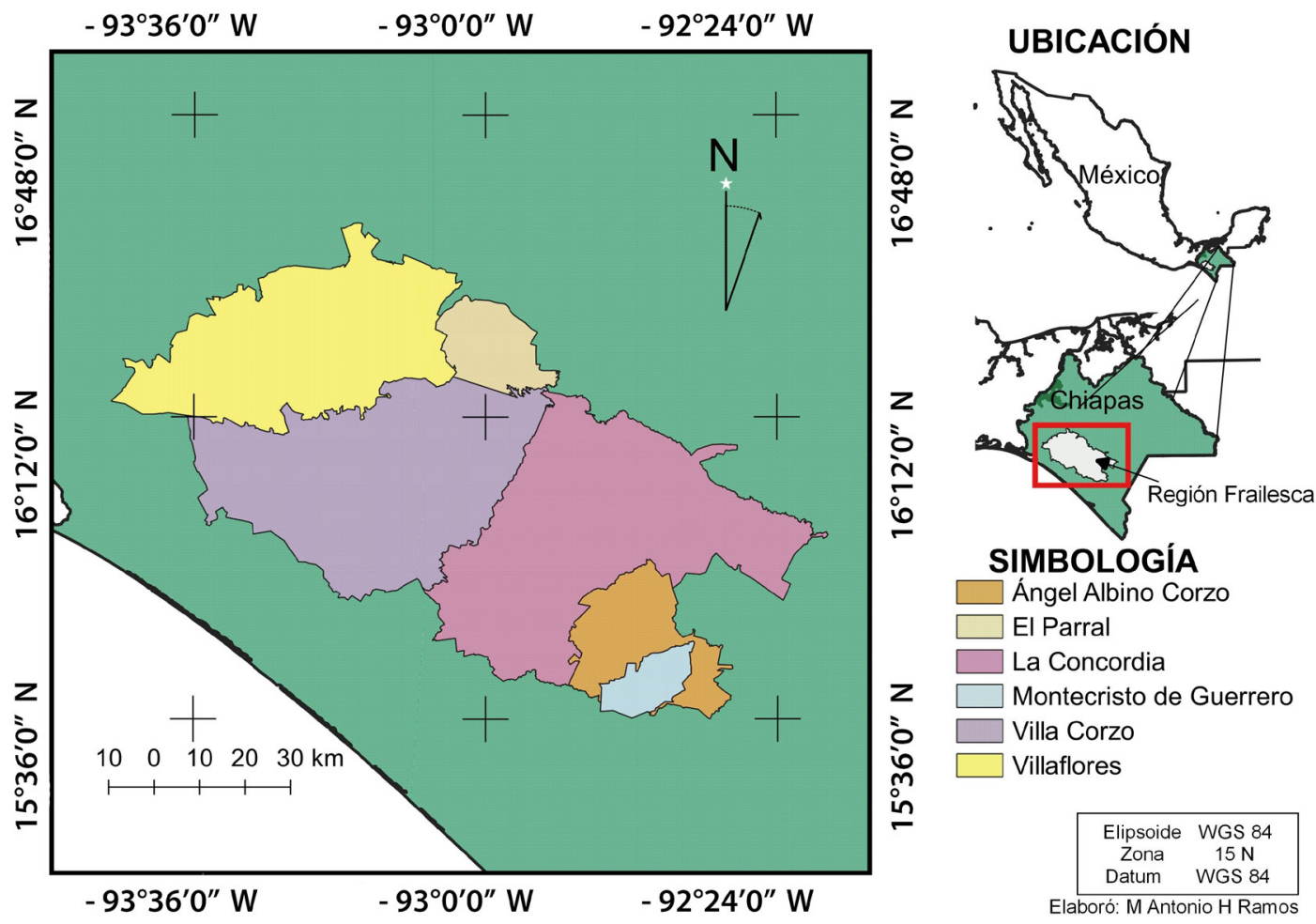

Figura 1. Ubicación geográfica de la región Frailesca, Chiapas.

Figure 1. Geographic location of the Frailesca region, Chiapas.

do, $3.5 \mathrm{~T} / \mathrm{ha}$; y el café, con el $6 \%$ de la producción del estado (SIAP, 2020). Además, en la última década se ha incrementado la actividad ganadera de doble propósito: mediante la mejora de los pastizales nativos o cultivados y la introducción de razas mejoradas (Campos y col., 2019). Prevalece también una diversidad de climas, así como costumbres y tradiciones que favorecen el cultivo de maíces locales en la región (Guevara y col., 2020).

\section{Obtención de la información}

En el periodo de marzo a diciembre de 2019 se recabó la información primaria a través de entrevistas semiestructuradas y observación participante, la cual se procesó y analizó entre enero y marzo de 2020. Para ello, se seleccionaron $80 \mathrm{UPF}$ de 20 comunidades (Tabla 1) mediante un muestreo por redes del tipo "no probabilístico" (Hernández y col., 2014), ya que se desconocía la población en lo tocante a cantidad y distribución de las UPF que cultivan maíces locales en la región. Los puntos de par- tida para el muestreo por redes fueron identificados en los Centros de Apoyo al Desarrollo Rural (CADER).

Las entrevistas semiestructuradas se aplicaron a los "jefes de familia" y constaron de 24 preguntas distribuidas en tres secciones: datos generales, capital humano y capital social. La guía de entrevista se diseñó a partir de la operacionalización de los capitales humano y social (Tabla 2) según el enfoque MVS propuesto por el Servicio Jesuita a Refugiados (SJR, 2017). Se consideraron 38 variables, 21 de ellas cuantitativas y 17 cualitativas. Para extraer, sintetizar y sistematizar la información de las entrevistas se utilizaron los métodos de interpretación de texto sugeridos por Hernández y col. (2014). Se desarrolló un proceso interactivo de consulta de expertos y validación en campo, para discriminar variables como "metros sobre el nivel mar" e incorporar otras como la cantidad de hijos fuera de la UPF que conservan los maíces locales. 
Tabla 1. Distribución por comunidades de jefes de UPF entrevistados en la Frailesca, Chiapas.

Table 1. Distribution by communities of FPU heads interviewed in the Frailesca, Chiapas.

\begin{tabular}{|l|c|c|c|c|c|}
\hline \multicolumn{1}{|c|}{ Comunidad } & $\begin{array}{c}\text { Cantidad } \\
\text { UPF* }\end{array}$ & Comunidad & $\begin{array}{c}\text { Cantidad } \\
\text { UPF }\end{array}$ & Comunidad & $\begin{array}{c}\text { Cantidad } \\
\text { UPF }\end{array}$ \\
\hline Guadalupe Victoria & 6 & Jesús M. Garza & 3 & Palenquelos Pinos & 3 \\
\hline Roblada Grande & 5 & Benito Juárez & 3 & Los Ángeles & 4 \\
\hline Villa Hermosa & 5 & Ranchería La Esperanza & 2 & Valle Morelos & 4 \\
\hline Buena Vista & 3 & Monterrey & 3 & Villa Corzo & 2 \\
\hline Juan Sabines & 3 & La Paz & 6 & Chiquinillal & 6 \\
\hline Francisco I. Madero & 5 & Jaltenango & 2 & San Nicolás & 4 \\
\hline Jerusalén & 5 & Libertad el Pajal & 6 & & \\
\hline
\end{tabular}

*UPF: Unidades de Producción Familiar.

\section{Análisis estadístico}

Las 21 variables cuantitativas (Tabla 2) se contemplaron para un análisis factorial por el método de componentes principales, con la finalidad de disminuir e integrar la cantidad de variables en dimensiones. De estas variables, fueron discriminadas las que mostraron un Coeficiente de Variación inferior al 10 \% (Tabla 3).

Se aplicó un análisis de conglomerados jerárquico mediante la distancia Euclediana y el método de vinculación de Ward. Para hacerlo, se usaron las dimensiones identificadas en el análisis de componentes principales (Tabla 3) de acuerdo con sus puntuaciones factoriales. Los tipos de UPF identificadas se validaron mediante las variables originales con un análisis de varianza para un modelo lineal. Para la comparación múltiple de media se recurrió a la prueba de Duncan.

Para las variables cualitativas se hicieron análisis de frecuencias y análisis exploratorios Factoriales de Correspondencias, que permitierondiscriminar las variables cualitativas no asociadas entre sí. Las correspondencias significativas se identificaron mediante la prueba de $\mathrm{Chi}^{2}$ y finalmente se revisaron las siguientes correspondencias:

- Tipo de conocimiento y formas de transmitirlo.

- Tipo de producto y su función (Insumo).

- Contratación de fuerza de trabajo y actividad productiva.

\section{RESULTADOS Y DISCUSIÓN}

Características socio-productivas de las UPF

Las 80 UPF estudiadas eran lideradas por hombres, en su mayoría de avanzada edad (59 años promedio, $\mathrm{DE}=10.17$ ) y bajo nivel escolar (primero de primaria). Las familias eran fundamentalmente nucleares e integradas en gran parte por 2 mujeres y 2 hombres (por lo general padres e hijos). Los 2 hijos podían ser menores de edad, dependientes de sus padres, o mayores de edad, solteros y/o divorciados que convivían en el hogar paterno y sus ingresos independientes contribuían, en alguna medida, a la economía familiar.

De acuerdo con la información obtenida, se evidenció una división sexual del trabajo dentro de las UPF que apuntaba a la vinculación directa de los hombres al trabajo agropecuario, mientras las mujeres se dedicaban a las actividades domésticas (Tabla 3), que incluyen el apoyo indirecto y no remunerado a la producción agropecuaria, atención a la familia, actividades de traspatio y desarrollo de pequeños negocios familiares.

Motivaciones para el cultivo y conservación de los maíces locales

Según lo referido por los agricultores, la conservación de los maíces locales responde a diversas motivaciones culturales, agronómicas y económicas, mientras que los maíces híbridos se fundamentan principalmente en las económicas. Sin embargo, es válido señalar que el 
Tabla 2. Variables analizadas en la caracterización.

Table 2. Variables analyzed during the characterization.

\begin{tabular}{|c|c|c|c|}
\hline Capital & Variables & $\begin{array}{l}\text { Tipo de } \\
\text { variable }\end{array}$ & Unidad de medida o categoría \\
\hline \multirow[t]{28}{*}{ Humano } & Edad & Cuantitativa & Años \\
\hline & Nivel escolar & Cualitativa & $\begin{array}{c}\text { Primaria, secundaria, preparatoria, } \\
\text { universidad }\end{array}$ \\
\hline & Estructura & Cuantitativa & Cantidad de miembros \\
\hline & Participación de hombres & Cuantitativa & Cantidad \\
\hline & $\begin{array}{l}\text { Hombres que cultivan maíces } \\
\text { locales }\end{array}$ & Cuantitativa & Cantidad \\
\hline & $\begin{array}{l}\text { Hombres que cultivan maíces } \\
\text { híbridos }\end{array}$ & Cuantitativa & Cantidad \\
\hline & $\begin{array}{l}\text { Hombres en otras actividades } \\
\text { agropecuarias }\end{array}$ & Cuantitativa & Cantidad \\
\hline & Otras actividades agropecuarias & Cualitativa & $\begin{array}{l}\text { Ganadería, cultivo de café, frijol, } \\
\text { frutales, otros }\end{array}$ \\
\hline & $\begin{array}{l}\text { Actividades agropecuarias y no } \\
\text { agropecuarias }\end{array}$ & Cuantitativa & Cantidad de personas \\
\hline & Trabajo asalariado & Cuantitativa & Cantidad de personas \\
\hline & Migración internacional & Cuantitativa & Cantidad de personas \\
\hline & Participación de mujeres & Cuantitativa & Cantidad \\
\hline & $\begin{array}{l}\text { Mujeres vinculadas al trabajo } \\
\text { reproductivo }\end{array}$ & Cuantitativa & Cantidad \\
\hline & Tiempo sembrando maíz & Cuantitativa & Años \\
\hline & Tiempo sembrando maíces locales & Cuantitativa & Años \\
\hline & Abandono del cultivo & Cualitativa & Sí, No \\
\hline & Tiempo de abandono & Cuantitativa & Cantidad de años \\
\hline & Total de hijos & Cuantitativa & Cantidad \\
\hline & $\begin{array}{c}\text { Hijos independizados de la UPF de } \\
\text { origen }\end{array}$ & Cuantitativa & Cantidad \\
\hline & $\begin{array}{l}\text { Hijos independizados de la UPF de } \\
\text { origen que conservan los maíces } \\
\text { locales }\end{array}$ & Cuantitativa & Cantidad \\
\hline & Contratación de fuerza de trabajo & Cualitativa & Sí, No \\
\hline & Actividad productiva & Cualitativa & $\begin{array}{l}\text { Actividad del ciclo productivo: limpia, } \\
\text { siembra, fertilización, cosecha, } \\
\text { beneficio } \\
\text { Causas de la contratación }\end{array}$ \\
\hline & $\begin{array}{l}\text { Motivaciones por el cultivo de maíz } \\
\text { local }\end{array}$ & Cualitativa & $\begin{array}{c}\text { Culturales, agronómicas, económicas, } \\
\text { otras }\end{array}$ \\
\hline & $\begin{array}{c}\text { Motivaciones por el cultivo de maíz } \\
\text { híbrido }\end{array}$ & Cualitativa & Culturales, agronómicas, económicas \\
\hline & Conservación de las semillas locales & Cualitativa & Sí, No \\
\hline & Tipos de conocimiento & Cualitativa & Familiar, local, externo, propio \\
\hline & $\begin{array}{l}\text { Formas de trasmisión del } \\
\text { conocimiento }\end{array}$ & Cualitativa & Vertical, horizontal, experiencia \\
\hline & Tipo de producto & Cualitativa & Químico, orgánico, ambos \\
\hline
\end{tabular}

Continúa... 


\begin{tabular}{|c|c|c|c|}
\hline & Función del producto (Insumo) & Cualitativa & Uso: herbicida, plaguicida y fertilizante \\
\hline \multirow[t]{9}{*}{ Social } & Relaciones locales & Cuantitativa & Cantidad \\
\hline & Tipo de relaciones de cooperación & Cualitativa & $\begin{array}{l}\text { Comunitarias: lazos vecinales, } \\
\text { familiares y compadrazgo. } \\
\text { Productivas: invitadito, cambio de } \\
\text { mano }\end{array}$ \\
\hline & Relaciones de cooperación & Cuantitativa & Cantidad de Relaciones \\
\hline & Participación en redes & Cualitativa & Sí, No \\
\hline & Forma de participación & Cualitativa & Formales, Informales \\
\hline & Relaciones institucionales & Cuantitativa & Cantidad \\
\hline & Tipo & Cualitativa & $\begin{array}{l}\text { Relaciones de subsidio, relaciones } \\
\text { comerciales, asesoría técnica }\end{array}$ \\
\hline & Relaciones de subsidio & Cuantitativa & Cantidad \\
\hline & Tipo de subsidios & Cualitativa & $\begin{array}{c}\text { Producción para el Bienestar, Prospera } \\
\text { y la Pensión para el Bienestar de las } \\
\text { Personas Adultas Mayores }\end{array}$ \\
\hline
\end{tabular}

Tabla 3. Componentes principales extraídas para la tipificación de UPF.

Table 3. Main components extracted for the typification of FPU.

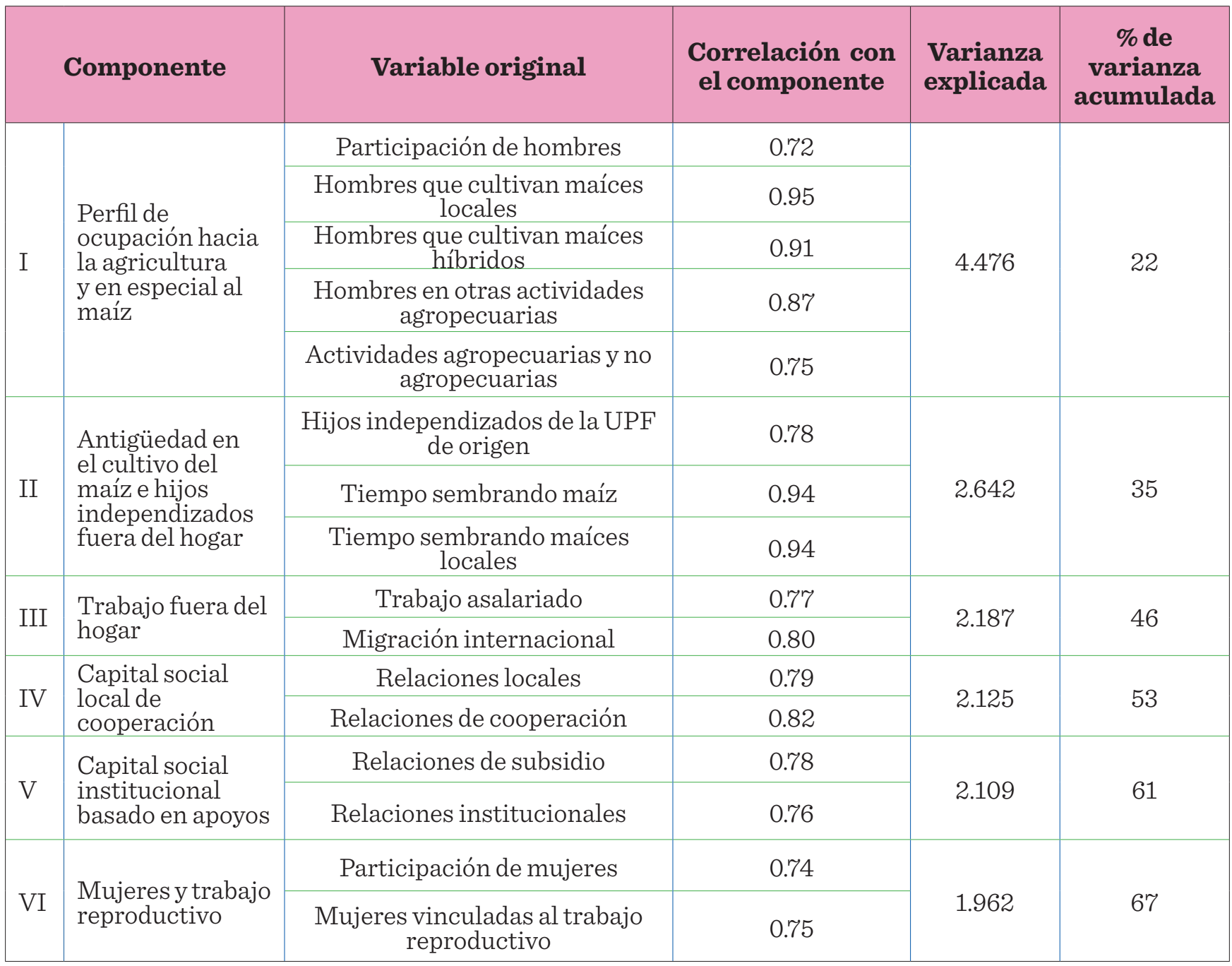


factor económico en el primer caso es con fines ahorrativos, pues se encontró que el $77 \%$ de los agricultores entrevistados seleccionaba y conservaba sus propias semillas con el propósito de usarlas en cultivos posteriores. Según Hernández y col. (2020), esto les permite disminuir los costos de producción, les genera independencia, autonomía, aprovechamiento de los recursos locales, identitarios y tradicionales. En el caso de los maíces híbridos, el móvil para su cultivo obedece al incremento de utilidades. En tal sentido, las 80 UPF cultivan un total de 103 ha de maíces locales y 148 de maíces híbridos.

Tipo de conocimiento y formas de transmitirlo Para transmitir el conocimiento se identificaron las categorías horizontal, vertical y experiencial, así como sus combinaciones. En cuanto al tipo de conocimiento se encontró al familiar, local, externo, propio y combinaciones entre ellos. En ese tenor, los agricultores, en su mayoría, reconocen que su conocimiento, en primera instancia, es de tipo familiar. También convergen otros, en mayor o menor medida, el local se origina entre los propios agricultores y el externo es el recibido de instituciones exógenas. Un grupo menor considera que es fruto de su propia experiencia. Esto significa que no predomina un solo tipo de conocimiento, sino que se integran aquellos heredados de generaciones precedentes (asociadas a los maíces locales) con las tecnologías modernas (asociadas a los maíces híbridos y paquetes tecnológicos) para conformar un diálogo de saberes que Guevara y col. (2018a) denominan una creación y recreación constante del conocimiento.

El análisis factorial de correspondencias identificó relaciones significativas $(P=0.000)$ entre el tipo de conocimiento y la forma en que se trasmite a partir de tres dimensiones (Figura 2). Las dimensiones 1 y 2 permiten asociar al conocimiento propio a la experiencia o vivencia personal, mientras que el familiar se relaciona con la transmisión vertical, pero incluye formas experienciales. Dentro de la misma generación, se detecta un tejido de relaciones que trascien- de el ámbito familiar e involucra a otros actores de la comunidad que comparten espacios similares pero experiencias más o menos diferentes. La dimensión 3 vincula al conocimiento local con formas horizontales de transmisión, lo que es recurrente con la idea del espacio físico comunitario, la milpa y las áreas sociales como escenarios de aprendizaje. Este conjunto de relaciones enriquece el proceso de aprendizaje en torno a los maíces locales.

\section{Tipo de producto y su función (Insumo)}

Con las estimaciones realizadas se detectó que el $95 \%$ de los productores aplicaba herbicidas, el $84 \%$ plaguicidas y el $88 \%$ fertilizantes, todos de tipo químico. El análisis factorial de correspondencias condujo a establecer una asociación significativa $(P=0.006)$ mediante dos dimensiones (Figura 3). La dimensión 1 (plano horizontal) mostró una tendencia minoritaria al uso de plaguicidas orgánicos. La dimensión 2 (vertical) arrojó una tendencia minoritaria al empleo combinado de compuestos orgánicos y químicos, que estuvo asociado con el uso de fertilizantes de ambos tipos. Esto concuerda con Martínez y col. (2020b), quienes plantearon que el $93 \%$ de los agricultores utiliza altas cantidades de agroquímicos. El $62 \%$ de los entrevistados de este estudio afirmó que suministraba a los maíces locales la misma cantidad de productos que a sus maíces híbridos. Es decir, que de acuerdo con Martínez-Aguilar (2021), las prácticas de manejo de los maíces (tanto locales como híbridos) actualmente contribuyen al deterioro de los recursos naturales de las UPF, como el suelo y el agua, y aumentan la dependencia externa por el uso de productos industriales.

\section{Contratación de fuerza de trabajo y actividad productiva}

Uno de los principales problemas que enfrentaban las UPF en cuanto al capital humano fue la insuficiente fuerza de trabajo familiar vinculada a las labores agropecuarias, lo que obligaba a ocupar trabajadores ajenos a la UPF para cubrir las actividades del ciclo productivo. En la Figura 4 se muestra la alta frecuencia de empleo de jornaleros, sobre todo en la 


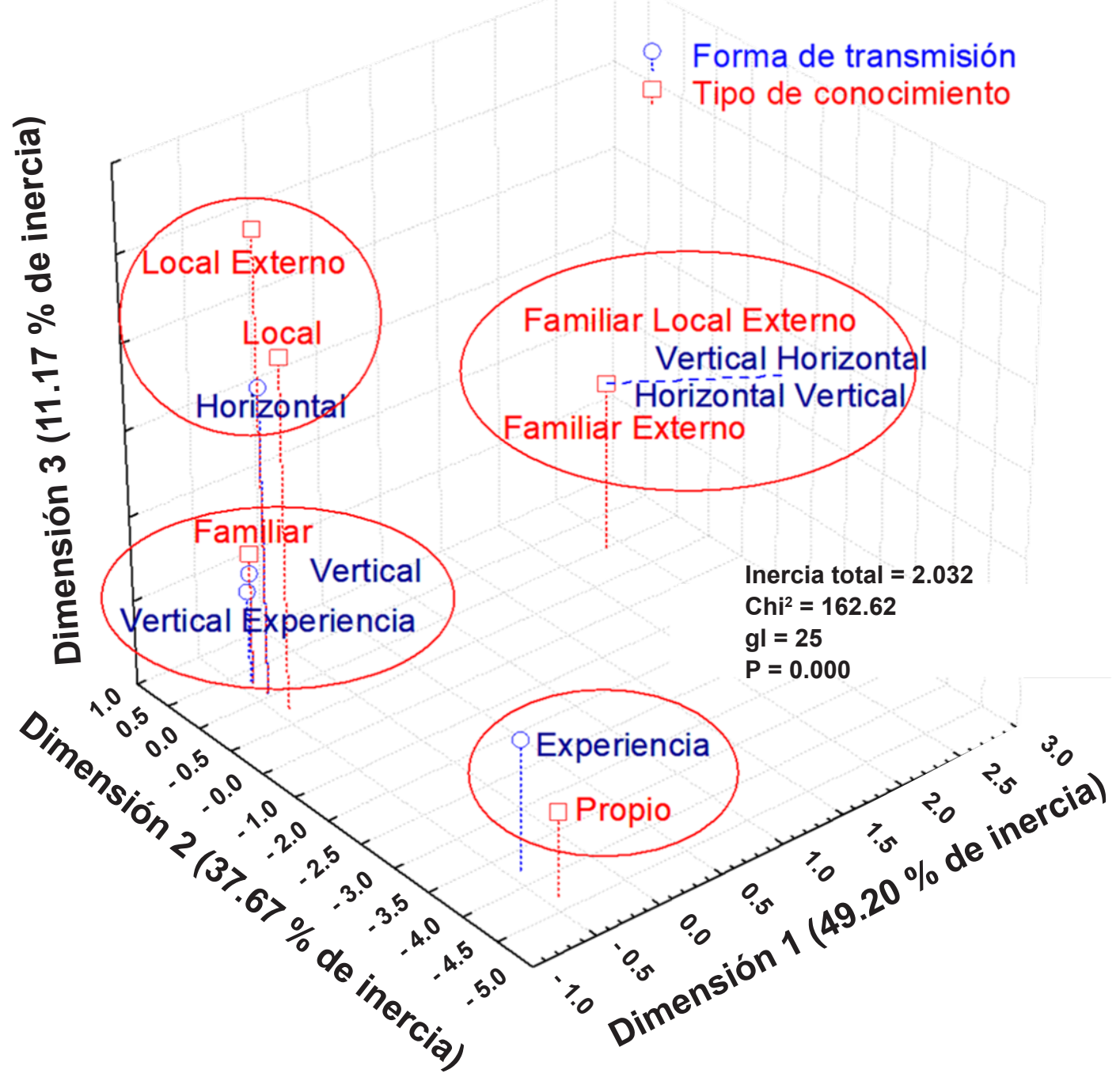

Figura 2. Tipo y formas de trasmisión del conocimiento en UPF, región Frailesca, Chiapas.

Figure 2. Type and forms of knowledge transmission at FPU in the Frailesca, Chiapas.

siembra, la fertilización y la cosecha, más no así en la limpia, ya que para ello el propio agricultor se valía de productos químicos aplicados con bombas aspersoras, lo que facilitaba su trabajo y disminuía los gastos de producción. La baja contratación en la cosecha del maíz obedecía a la renta de maquinaria para desgranar y a escasas manifestaciones de cooperación local.

\section{Participación en redes y organizaciones}

La participación en redes, como elemento del capital social, no aportó a la tipificación de las UPF, pero contribuyó a la caracterización general. Al respecto, la conformación o partici- pación en organizaciones para la producción de maíz no es común en las UPF estudiadas. Sólo en el $8 \%$ de ellas existe algún tipo de afiliación formal a organizaciones que apoyen el desarrollo de la producción. En concordancia con Campos y col. (2019), tampoco se destacaron redes formales con instituciones para la demanda de asesorías técnicas.

Tipos de UPF que cultivan maíces locales Mediante el análisis factorial de Componentes Principales se encontró que la diversidad de características de estas UPF se manifiesta en 6 dimensiones, que explican el $67 \%$ de la variabilidad total (Tabla 3). Estas dimensio- 


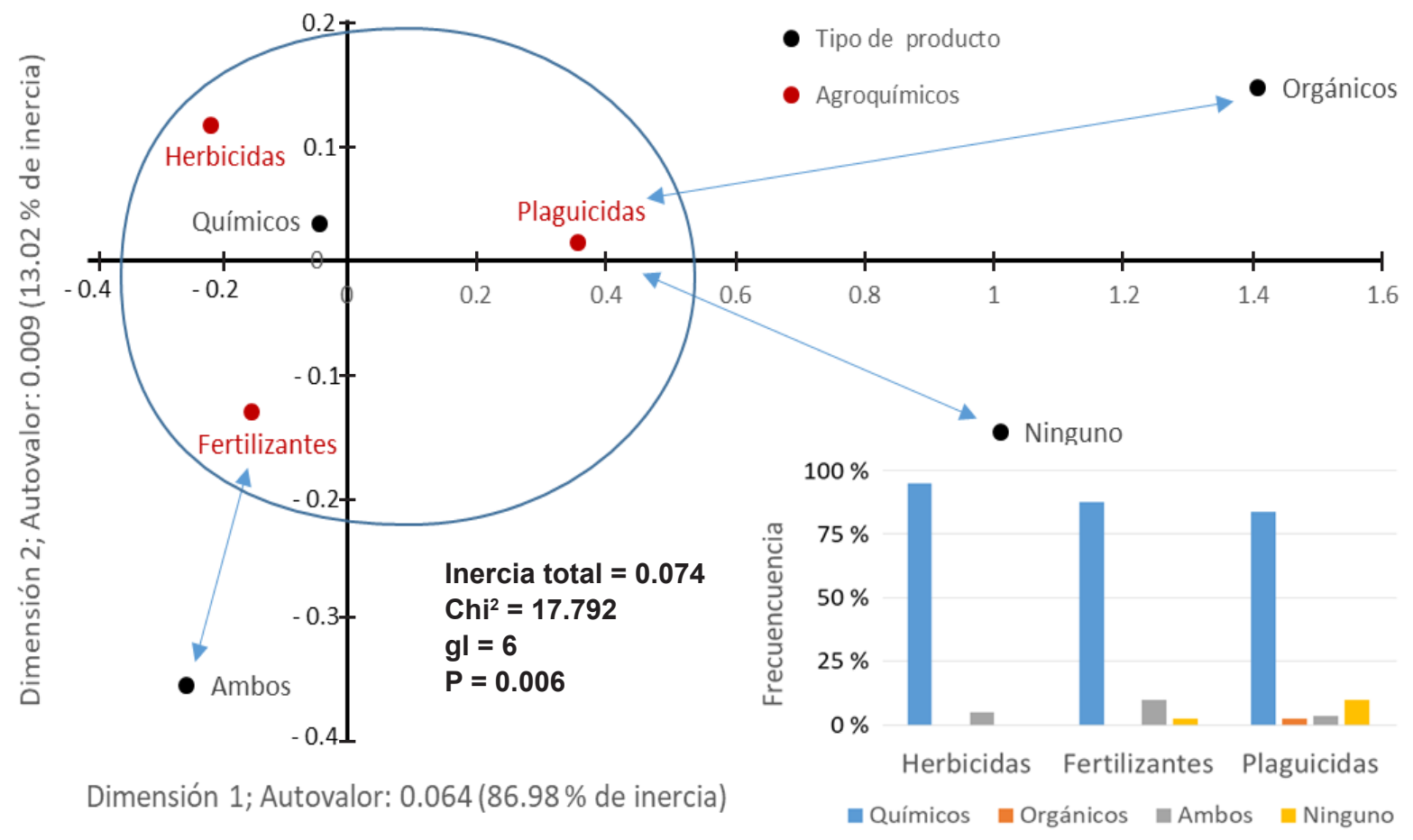

Figura 3. Asociaciones entre actividades del ciclo de producción del maíz local y los productos utilizados.

Figure 3. Associations between activities of the local maize production cycle and the used products.

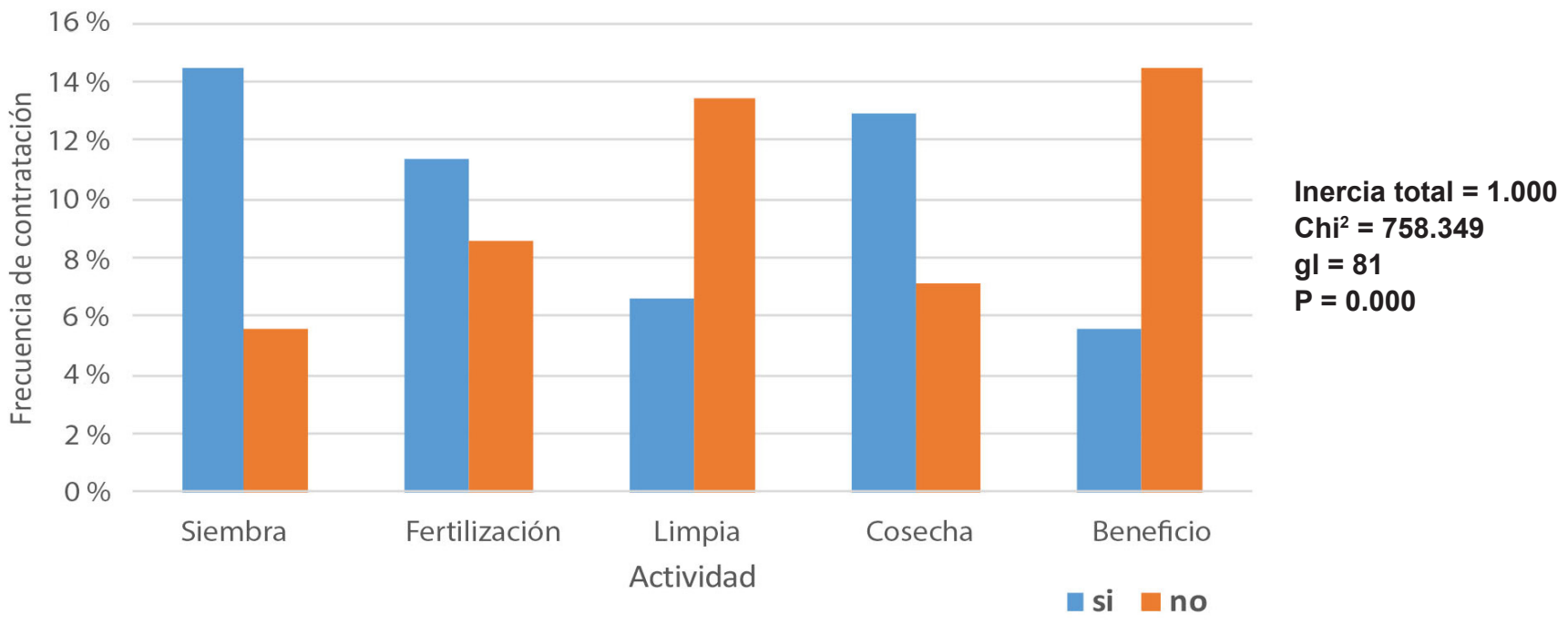

Figura 4. Contratación de fuerza de trabajo por actividades del ciclo productivo de las UPF.

Figure 4. Hiring of workforce by activity during the productive cycle at FPU.

nes se etiquetaron, según sus correlaciones con las variables originales, de la siguiente forma:

- Perfil de ocupación hacia la agricultura y en especial hacia el maíz

- Antigüedad en el cultivo del maíz e hijos independizados de la UPF de origen

- Trabajo fuera del hogar 
- Capital social local de cooperación

- Capital social institucional basado en apoyos

- Mujeres y trabajo reproductivo

Dichas dimensiones son la base de la identificación de tipos de UPF en este contexto productivo. Básicamente, las estructuras socioproductivas no son homogéneas y el análisis de conglomerados permitió identificar 6 tipos de UPF (Figura 5). Los 6 grupos fueron examinados para definir su comportamiento referente a los componentes de la Tabla 3 y los resultados se muestran en la Figura 6.

Perfil de ocupación hacia la agricultura y en especial hacia el maíz

En el componente I, el tercer grupo (G3) presentó mejor perfil de ocupación hacia la agricultura y en especial al maíz (Figura 6). En general, hay una mayor participación familiar en las actividades agropecuarias de la UPF y en particular en el cultivo de los maíces locales, con 2 hombres como promedio (Tabla 4). El resto de los grupos, G1, G2, G4, G5 y G6 difícilmente contaba con el apoyo de un solo hombre en labores agropecuarias (cultivo de maíz) y no agropecuarias, lo que corrobora la necesidad de contratación de mano de obra y, por consiguiente, mayor dependencia de factores externos.

Antigüedad en el cultivo del maíz e hijos independizados fuera del hogar

En el componente II, con 3 variables asociadas (Tabla 4), se observó que los G1, G2 y G3 eran los agricultores con mayor antigüedad en la conservación del maíz local, con 62.3, 42.7 y 47.3 años, respectivamente. Por tanto, estos grupos acumulaban también mayor experiencia y sentido de pertenencia por los maíces locales, aun cuando los agricultores del G3 manifestaron que dejaron temporalmente (al menos por un año) de sembrarlos. Esta información se corroboró con el cálculo de la diferencia entre los años que cultivaron maíces en general y los que cultivaron maíces locales particularmente.

Los G1, G3 y G5 presentaron mayor cantidad de hijos independientes de las UPF de origen,

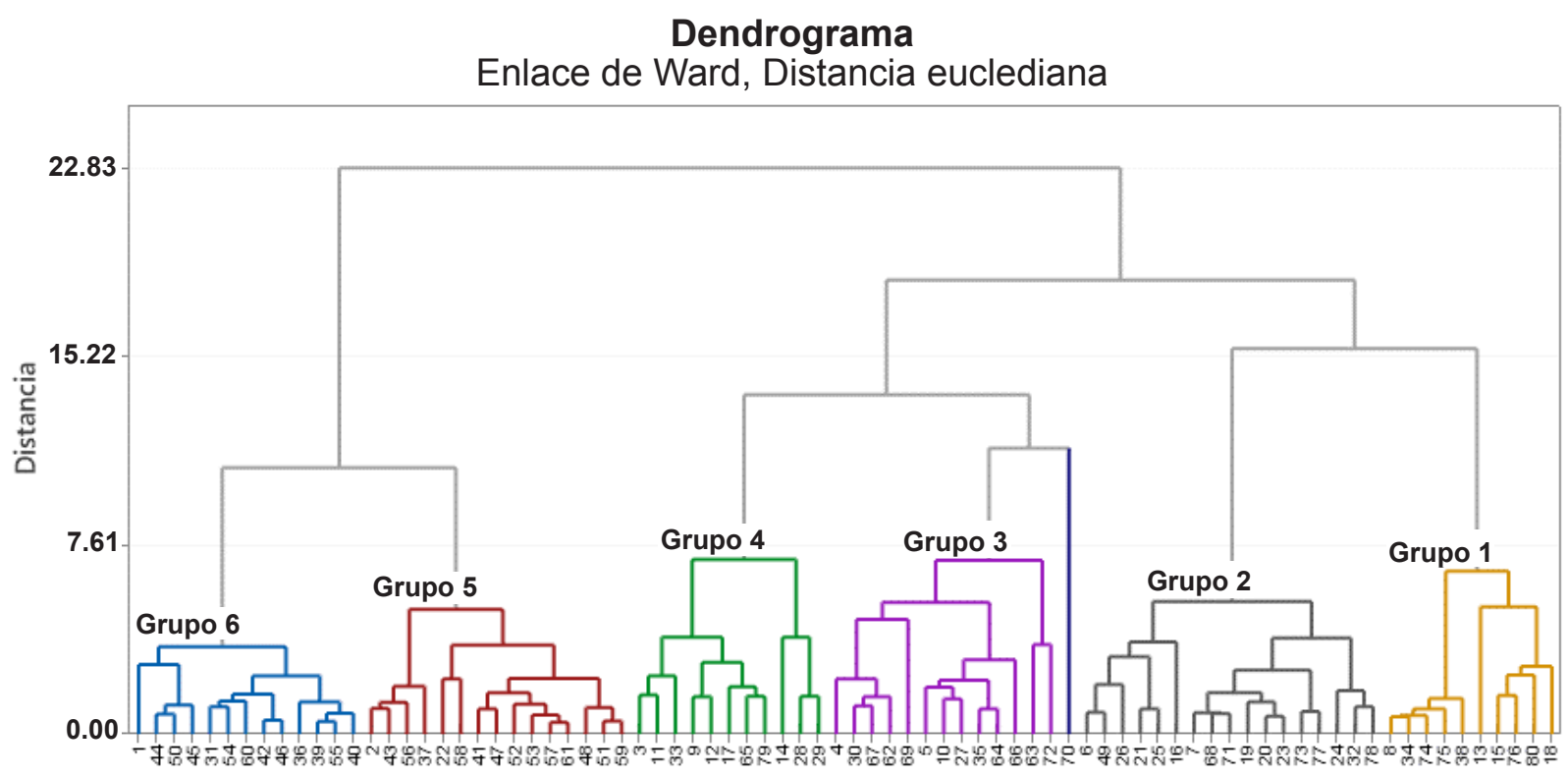

Observaciones (Unidades de Producción Familiar)

Figura 5. Diagrama de tipología en cuanto a los capitales humano y social de UPF de maíces locales en la Frailesca, Chiapas.

Figure 5. Typology diagram regarding FPU's human and social capitals of local maize in la Frailesca, Chiapas. 


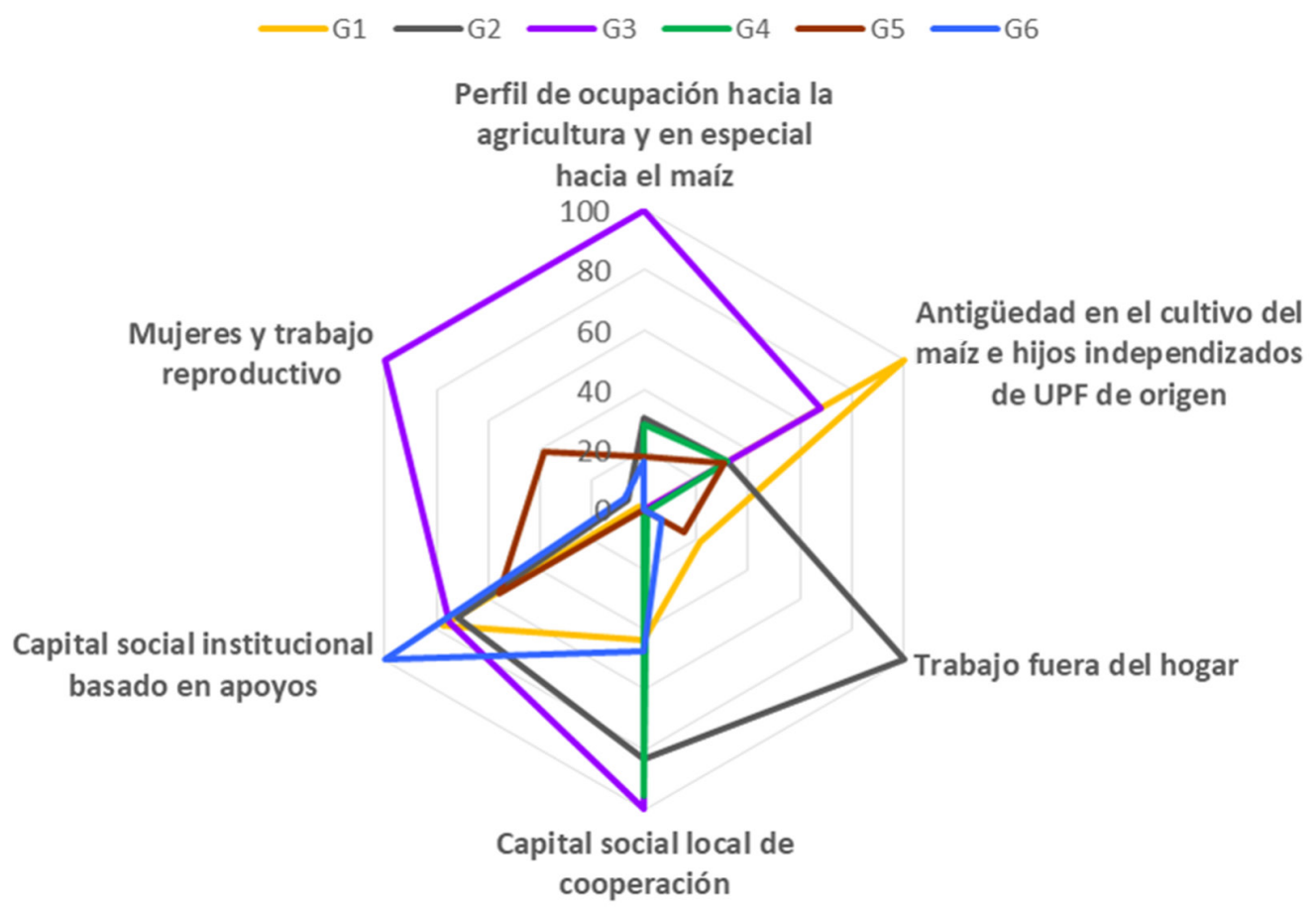

Figura 6. Caracterización de los grupos identificados a partir de las componentes de UPF con maíces locales, la Frailesca, Chiapas.

Figure 6. Characterization of identified groups from the components of local maize FPU's in la Frailesca, Chiapas.

con 5, 4 y 3, respectivamente. Sin embargo, mediante el análisis de contenido se demostró que esto no garantiza la conservación de los maíces locales, pues sólo en el $31 \%$ de los casos los hijos, al independizarse de la UPF de origen, mantenían el cultivo de maíces locales. En ese tenor, se identificaron 15 UPF con 1 hijo independizado de la UPF que conserva el cultivo de los maíces locales, $6 \mathrm{UPF}$ con 2 , 2 UPF con 3 y 1 UPF con 4 . Por tanto, de acuerdo con Guevara y col. (2020), la edad avanzada de los agricultores no representa una problemática en sí, toda vez que es un fenómeno natural, lo que realmente compromete la continuidad de la conservación de los maíces locales en el mediano y largo plazo es el insuficiente reemplazo generacional en la actividad.

\section{Trabajo fuera del hogar}

El G2 mostró las puntuaciones más altas dentro de la componente III (Tabla 4). El trabajo fuera del hogar, que contempla el trabajo asalariado y la migración internacional en este grupo, tuvo mayor significación que en el resto, lo que indica la existencia de estrategias más diversificadas fuera del sector agropecuario que complementan los ingresos familiares. Los G1, G3, G4, G5 y G6 no muestran índices significativos de ofrecer su fuerza de trabajo al sector secundario o terciario (como asalariados), por tanto, sus estrategias se orientan más hacia la especialización en el sector primario, en contraposición a lo encontrado en otros estudios (Cadena-Iñiguez y col., 2016).

\section{Capital social local de cooperación}

El Componente IV (Tabla 4) revela que los G2, G3, G4 y G6 son los que mantienen mayor cantidad de relaciones locales. Sin embargo, las de los G2, G3 y G4 trascienden el ámbito local. En sentido general, se encontró que en todos los grupos predominan las relaciones locales y de cooperación a través de la solidaridadentre vecinos, familiares y compadres, que son propias de la agricultura familiar. Sin embargo, los G2, G3 y G4 conservan prác- 
Tabla 4. Estadígrafos de tendencia central y de dispersión de las variables que caracterizan a las diferentes UPF de maíces locales.

Table 4. Statistics of central tendency and dispersion of the variables that characterize the different FPU typologies of local maize.

\begin{tabular}{|c|c|c|c|c|c|c|c|c|c|c|c|c|c|}
\hline \multicolumn{14}{|c|}{ Componente I: Perfil de ocupación hacia la agricultura y en especial al maíz } \\
\hline \multirow{3}{*}{$\begin{array}{l}\text { Variables } \\
\text { originales/ } \\
\text { unidades de } \\
\text { medidas }\end{array}$} & \multirow{2}{*}{\multicolumn{2}{|c|}{$\frac{\text { G1 }}{17 \text { familias* }^{*}}$}} & \multirow{2}{*}{\multicolumn{2}{|c|}{$\begin{array}{c}\text { G2 } \\
\text { familias }\end{array}$}} & \multirow{2}{*}{\multicolumn{2}{|c|}{$\frac{\text { G3 }}{13 \text { familias }}$}} & \multirow{2}{*}{\multicolumn{2}{|c|}{$\frac{\text { G4 }}{1 \text { familias }}$}} & \multirow{2}{*}{\multicolumn{2}{|c|}{$\begin{array}{c}\text { G5 } \\
15 \text { familias }\end{array}$}} & \multirow{2}{*}{\multicolumn{2}{|c|}{$\begin{array}{c}\text { G6 } \\
13 \text { familias }\end{array}$}} & \multirow{3}{*}{ Sig } \\
\hline & & & & & & & & & & & & & \\
\hline & $\mathrm{X}$ & $\mathrm{DE}$ & $\mathrm{X}$ & $\mathrm{DE}$ & $\mathrm{X}$ & $\mathrm{DE}$ & $\mathrm{X}$ & $\mathrm{DE}$ & $\mathrm{X}$ & $\mathrm{DE}$ & $\mathrm{X}$ & $\mathrm{DE}$ & \\
\hline $\begin{array}{l}\text { Participación de } \\
\text { hombres }\end{array}$ & $1.5^{\mathrm{c}}$ & 0.717 & $2.8^{\mathrm{a}}$ & 1.03 & $2.6^{\mathrm{ba}}$ & 1.446 & $2.4^{\mathrm{ba}}$ & 1.286 & $2.1^{\mathrm{bac}}$ & 0.799 & $1.8^{\mathrm{bc}}$ & 0.899 & 0.013 \\
\hline $\begin{array}{l}\text { Hombres que } \\
\text { cultivan maíces } \\
\text { locales }\end{array}$ & $1.3^{b}$ & 0.47 & $1.4^{\mathrm{b}}$ & 0.52 & $2.0^{\mathrm{a}}$ & 1.155 & $1.5^{\mathrm{b}}$ & 0.688 & $1.3^{b}$ & 0.488 & $1.4^{b}$ & 0.65 & 0.098 \\
\hline $\begin{array}{l}\text { Hombres que } \\
\text { cultivan maíces } \\
\text { híbridos }\end{array}$ & $0.9^{b}$ & 0.748 & $1.3^{\mathrm{ab}}$ & 0.67 & $1.8^{\mathrm{a}}$ & 1.235 & $1.3^{\mathrm{ab}}$ & 0.905 & $1.3^{\mathrm{ab}}$ & 0.594 & $1.3^{\mathrm{ab}}$ & 0.751 & 0.218 \\
\hline $\begin{array}{l}\text { Hombres en } \\
\text { otras actividades } \\
\text { agropecuarias }\end{array}$ & 1.3 & 0.849 & 1.2 & 0.63 & 1.9 & 1.441 & 1.4 & 0.505 & 1.3 & 0.594 & 1.2 & 0.439 & 0.235 \\
\hline $\begin{array}{l}\text { Actividades } \\
\text { agropecuarias y } \\
\text { no agropecuarias }\end{array}$ & $0.2^{c}$ & 0.562 & $0.9^{\mathrm{ba}}$ & 0.57 & $1.2^{\mathrm{a}}$ & 1.166 & $0.5^{\mathrm{cb}}$ & 0.522 & $0.3^{c}$ & 0.488 & $0.7^{c b a}$ & 0.855 & 0.006 \\
\hline \multicolumn{14}{|c|}{ Componente II: Antigüedad en el cultivo del maíz e hijos fuera del hogar } \\
\hline & \multicolumn{2}{|c|}{ G1 } & \multicolumn{2}{|c|}{ G2 } & \multicolumn{2}{|c|}{ G3 } & \multicolumn{2}{|c|}{ G4 } & \multicolumn{2}{|c|}{ G5 } & \multicolumn{2}{|c|}{ G6 } & \multirow{3}{*}{ Sig } \\
\hline & \multicolumn{2}{|c|}{17 familias } & \multicolumn{2}{|c|}{10 familias } & \multicolumn{2}{|c|}{13 familias } & \multicolumn{2}{|c|}{11 familias } & \multicolumn{2}{|c|}{15 familias } & \multicolumn{2}{|c|}{13 familias } & \\
\hline & $\mathrm{X}$ & $\mathrm{DE}$ & $\mathrm{X}$ & $\mathrm{DE}$ & $\mathrm{X}$ & $\mathrm{DE}$ & $\mathrm{X}$ & $\mathrm{DE}$ & $\mathrm{X}$ & $\mathrm{DE}$ & $\mathrm{X}$ & $\mathrm{DE}$ & \\
\hline $\begin{array}{l}\text { Hijos } \\
\text { independizados } \\
\text { de la UPF de } \\
\text { origen }\end{array}$ & $5.4^{\mathrm{a}}$ & 2.21 & $1.2^{\mathrm{c}}$ & 1.62 & $4.2^{\mathrm{ba}}$ & 3.032 & $1.4^{\mathrm{c}}$ & 2.111 & $2.7^{\mathrm{cb}}$ & 1.163 & $1.6^{\mathrm{c}}$ & 0.87 & 0 \\
\hline $\begin{array}{l}\text { Tiempo } \\
\text { sembrando maíz }\end{array}$ & $62.3^{\mathrm{a}}$ & 9.912 & $42.7^{\mathrm{b}}$ & 17 & $48.4^{\mathrm{b}}$ & 14.69 & $41.3^{b}$ & 18.06 & $32.1^{\mathrm{dc}}$ & 6.468 & $22.4^{\mathrm{d}}$ & 7.148 & 0 \\
\hline $\begin{array}{l}\text { Tiempo } \\
\text { sembrando } \\
\text { maíces locales }\end{array}$ & $62.3^{\mathrm{a}}$ & 9.912 & $42.7^{\mathrm{b}}$ & 17 & $47.3^{b}$ & 14.81 & $40.7^{b}$ & 19.2 & $32.1^{\mathrm{dc}}$ & 6.468 & $22.4^{\mathrm{d}}$ & 7.148 & 0 \\
\hline \multicolumn{14}{|c|}{ Componente III: Trabajo fuera del hogar } \\
\hline & \multicolumn{2}{|c|}{ G1 } & \multicolumn{2}{|c|}{ G2 } & \multicolumn{2}{|c|}{ G3 } & \multicolumn{2}{|c|}{ G4 } & \multicolumn{2}{|c|}{ G5 } & G & 46 & \\
\hline & $17 \mathrm{fa}$ & milias & $10 \mathrm{far}$ & nilias & $13 \mathrm{far}$ & nilias & $11 \mathrm{far}$ & nilias & $15 \mathrm{far}$ & nilias & $13 \mathrm{far}$ & milias & Sig \\
\hline & $X$ & $\mathrm{DE}$ & $X$ & $\mathrm{DE}$ & $X$ & $\mathrm{DE}$ & $X$ & $\mathrm{DE}$ & $X$ & $\mathrm{DE}$ & $X$ & $\mathrm{DE}$ & \\
\hline $\begin{array}{l}\text { Trabajo } \\
\text { asalariado }\end{array}$ & $0.6^{b}$ & 0.996 & $1.6^{\mathrm{a}}$ & 1.51 & $0.2^{b}$ & 0.555 & $0.2^{\mathrm{b}}$ & 0.405 & $0.4^{b}$ & 0.507 & $0.1^{b}$ & 0.277 & 0.000 \\
\hline $\begin{array}{l}\text { Migración } \\
\text { internacional }\end{array}$ & $0^{\mathrm{b}}$ & 0 & $0.7^{a}$ & 0.68 & $0^{b}$ & 0 & $0^{b}$ & 0 & $0^{\mathrm{b}}$ & 0 & $\mathrm{O}^{\mathrm{b}}$ & 0 & 0 \\
\hline & & om & ente & V: C & 0 & cial & 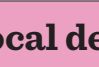 & 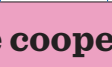 & ació & & & & \\
\hline & & 41 & C & & G & 3 & & 4 & G & 5 & G & 46 & \\
\hline & $17 \mathrm{fa}$ & milias & $10 \mathrm{far}$ & nilias & $13 \mathrm{far}$ & nilias & $11 \mathrm{far}$ & nilias & $15 \mathrm{far}$ & nilias & $13 \mathrm{far}$ & milias & Sig \\
\hline & $X$ & $\mathrm{DE}$ & $X$ & $\mathrm{DE}$ & $X$ & $\mathrm{DE}$ & $X$ & $\mathrm{DE}$ & $X$ & $\mathrm{DE}$ & $\mathrm{X}$ & $\mathrm{DE}$ & \\
\hline $\begin{array}{l}\text { Relaciones } \\
\text { locales }\end{array}$ & $4.2^{\mathrm{bc}}$ & 1.015 & $4.8^{\mathrm{a}}$ & 0.28 & $4.9^{\mathrm{a}}$ & 0.277 & $4.6^{\mathrm{ab}}$ & 0.674 & $3.7^{c}$ & 0.799 & $4.6^{\mathrm{ab}}$ & 0.506 & 0.000 \\
\hline
\end{tabular}




\begin{tabular}{|c|c|c|c|c|c|c|c|c|c|c|c|c|c|}
\hline $\begin{array}{l}\text { Relaciones de } \\
\text { cooperación }\end{array}$ & $4.2^{\mathrm{bc}}$ & 1.3 & $5.2^{\mathrm{a}}$ & 0.63 & $4.9^{\mathrm{ba}}$ & 0.277 & $4.8^{\mathrm{ba}}$ & 0.874 & $3.3^{d}$ & 0.594 & $3.6^{\mathrm{dc}}$ & 0.768 & 0 \\
\hline \multicolumn{14}{|c|}{ Componente V: Capital social institucional basado en apoyos } \\
\hline & \multicolumn{2}{|c|}{ G1 } & \multicolumn{2}{|c|}{ G2 } & \multicolumn{2}{|c|}{ G3 } & \multicolumn{2}{|c|}{ G4 } & \multicolumn{2}{|c|}{ G5 } & \multicolumn{2}{|c|}{ G6 } & \multirow{3}{*}{ Sig } \\
\hline & \multicolumn{2}{|c|}{17 familias } & \multicolumn{2}{|c|}{10 familias } & \multicolumn{2}{|c|}{13 familias } & \multicolumn{2}{|c|}{11 familias } & \multicolumn{2}{|c|}{15 familias } & \multicolumn{2}{|c|}{13 familias } & \\
\hline & $\mathrm{X}$ & $\mathrm{DE}$ & $X$ & $\mathrm{DE}$ & $\mathrm{X}$ & $\mathrm{DE}$ & $\mathrm{X}$ & $\mathrm{DE}$ & $\mathrm{X}$ & $\mathrm{DE}$ & $\mathrm{X}$ & $\mathrm{DE}$ & \\
\hline $\begin{array}{l}\text { Relaciones de } \\
\text { subsidio }\end{array}$ & $0.9^{\mathrm{a}}$ & 0.243 & $0.9^{\mathrm{a}}$ & 0.57 & $1.1^{\mathrm{a}}$ & 0.277 & $0.4^{\mathrm{b}}$ & 0.505 & $0.9^{\mathrm{a}}$ & 0.258 & $1.1^{\mathrm{a}}$ & 0.555 & 0.000 \\
\hline $\begin{array}{l}\text { Relaciones } \\
\text { institucionales }\end{array}$ & $3.1^{\mathrm{a}}$ & 0.485 & $3.0^{\mathrm{a}}$ & 0.47 & $3.1^{\mathrm{a}}$ & 0.76 & $2.3^{b}$ & 0.786 & $2.3^{b}$ & 0.488 & $3.4^{\mathrm{a}}$ & 0.65 & 0.000 \\
\hline \multicolumn{14}{|c|}{ Componente VI: Mujeres y trabajo reproductivo } \\
\hline & \multicolumn{2}{|c|}{ G1 } & \multicolumn{2}{|c|}{ G2 } & \multicolumn{2}{|c|}{ G3 } & \multirow{2}{*}{\multicolumn{2}{|c|}{\begin{tabular}{c|c}
$\mathrm{G} 4$ \\
$11 f_{\text {amiliog }}$
\end{tabular}}} & \multirow{2}{*}{\multicolumn{2}{|c|}{ G5 }} & \multicolumn{2}{|c|}{ G6 } & \multirow{3}{*}{ Sig } \\
\hline & \multicolumn{2}{|c|}{17 familias } & \multicolumn{2}{|c|}{10 familias } & \multicolumn{2}{|c|}{13 familias } & & & & & \multicolumn{2}{|c|}{13 familias } & \\
\hline & $\mathrm{X}$ & $\mathrm{DE}$ & $\mathrm{X}$ & $\mathrm{DE}$ & $\mathrm{X}$ & $\mathrm{DE}$ & $\mathrm{X}$ & $\mathrm{DE}$ & $\mathrm{X}$ & $\mathrm{DE}$ & $\mathrm{X}$ & $\mathrm{DE}$ & \\
\hline $\begin{array}{l}\text { Participación de } \\
\text { mujeres }\end{array}$ & $1.3^{\mathrm{c}}$ & 0.786 & $2.4^{\mathrm{ab}}$ & 1.17 & $2.8^{\mathrm{a}}$ & 1.625 & $1.7^{\mathrm{cb}}$ & 1.009 & $2.8^{\mathrm{a}}$ & 1.32 & $2.1^{\text {acb }}$ & 0.899 & 0.003 \\
\hline $\begin{array}{l}\text { Mujeres } \\
\text { vinculadas } \\
\text { al trabajo } \\
\text { reproductivo }\end{array}$ & $1.0^{\mathrm{c}}$ & 0 & $1.0^{c}$ & 0 & $2.1^{\mathrm{a}}$ & 0.899 & $1.5^{\mathrm{b}}$ & 0.688 & $1.0^{c}$ & 0 & $1.0^{c}$ & 0.277 & 0 \\
\hline
\end{tabular}

${ }^{*}$ G1: grupo 1. Letras diferentes por fila indican diferencia significativa al aplicar la prueba de DUNCAN para $\mathrm{P}<0.05$. $\mathrm{X}=$ Media; DE = Desviación estándar; Sig. = Significación. ${ }^{* *}$ Una UPF no se incluyó en el análisis de varianza.

ticas de colaboración en el ámbito del trabajo agropecuario, como el "el invitadito" y "el cambio de mano". Mediante ellas, los agricultores se apoyan mutuamente en diferentes etapas del ciclo productivo sin remuneración alguna Martínez-Aguilar (2021). Según manifestaron los agricultores, estas formas de colaboración ya no constituyen una generalidad, pues han sido sustituidas por el uso de desgranadoras mecánicas, cuyo uso es común en la región.

Capital social institucional basado en apoyos El G6 presenta el mayor número de relaciones institucionales, toda vez que reconoce la asesoría técnica de instituciones como la Universidad Autónoma de Chiapas (UNACH), particularmente con la Facultad de Ciencias Agronómicas. Sin embargo, un $92 \%$ de los agricultores manifestó no contar con este tipo de conexiones. Por este motivo, las relaciones institucionales se basan fundamentalmente en las de carácter comercial, a través de organismos proveedores de insumos agropecuarios y los compradores de maíces, así como los de subsidios gubernamentales. En la Tabla 4 se muestra que los G1, G2, G3, G5 y G6 reciben al menos un sub- sidio gubernamental. Estos corresponden a subsidios para la familia como PROSPERA y la Pensión para el Bienestar de las Personas Adultas Mayores y para el cultivo de maíz como PROCAMPO (es la forma en que lo reconocen los productores, actualmente es el Programa de Producción para el Bienestar de la Subsecretaría de Alimentación y Competitividad, dependiente de la Secretaría de Agricultura y Desarrollo Rural). El programa consiste en el apoyo económico directo, sin intermediarios, a pequeños y medianos productores de granos, amaranto, chía, caña de azúcar, café y recientemente se incluyen los productores de cacao y miel de abeja. En 2021 se incrementó el respaldo a productores de granos con menos de 5 ha de temporal a \$ 2000 pesos por ha, y de más de 5 ha hasta 20 ha con $\$ 2200$ pesos por ha.

De acuerdo con la información de los agricultores, aunque no son suficientes los subsidios, complementan los costos de producción que las propias ganancias por concepto de maíces locales no logran sufragar. No obstante, de acuerdo con Bartra (2020), los subsidios pueden desestimular la diversificación de actividades 
y cultivos. Se pueden limitar así las capacidades para desarrollar una mayor eficiencia en la gestión de la UPF y de su economía.

\section{Mujeres y trabajo reproductivo}

En el G3 se presenta una mayor participación de la mujer en el trabajo reproductivo de la $\mathrm{UPF}$, con 2 mujeres como promedio, mientras el resto de los grupos solo cuenta con 1. En el trabajo reproductivo se contemplaron todas aquellas actividades que directa o indirectamente apoyan el proceso agropecuario y el funcionamiento de la UPF en sentido general, tales como la atención al traspatio familiar, las labores domésticas y el cuidado de los hijos. Estas se analizaron a través de la cantidad de mujeres que se vinculan directamente con ellas.

\section{Discusión}

La edad avanzada de los líderes de las familias es clave en la conservación de los maíces locales, ya que provee a las UPF de mayor experiencia y confianza para el cultivo, conseguidas como parte del proceso de transferencia vertical del conocimiento familiar de una generación a otra y enriquecidas con las nuevas tecnologías que se integran en la actualidad. Aunado a esto, es evidente el interés de los jóvenes por otras actividades más rentables, como la ganadería, los maíces híbridos y el café, lo que, de acuerdo con Guevara y col. (2018a; 2020), genera incertidumbre en la continuidad del cultivo de los maíces locales en estas UPF.

El excesivo uso de agroquímicos afecta los recursos naturales como el suelo, el agua y la diversidad de especies en los agroecosistemas de maíces locales y maíz en general de la Frailesca (Aguilar y col., 2019). Este uso indiscriminado y descontrolado de agroquímicos incide, además, según Martínez y col. (2020a), en los costos de producción, que son cada vez más elevados, a lo que se suma la necesidad de contratar fuerza de trabajo externa a la UPF y la baja conformación de redes que apoyen la producción de forma solidaria. Estos aspectos incrementan a su vez los niveles de dependencia externa de las UPF.
En cuanto a los tipos de UPF, el G3 se destaca por presentar una mayor participación familiar masculina, fundamental para el funcionamiento de la unidad de producción. El G1 es el más experimentado en el cultivo de los maíces locales, seguido de los G3 y G2. El G2 ha logrado mayor diversificación al presentar indicadores más significativos en la combinación del quehacer agropecuario con el trabajo asalariado y la migración internacional (Tabla 4). De acuerdo con Toledo (2008), esto le confiere mayor resiliencia ante eventos inesperados, mientras que el resto de los grupos son más vulnerables frente a tales circunstancias. Los G2, G3 y G4 desarrollan más las relaciones locales y de cooperación, mientras que el G6 las relaciones institucionales. El G4 recibe la menor cantidad de subsidios del gobierno porque no cumple los requisitos que exige el Programa Producción para el Bienestar, y probablemente ha desarrollado mayor autosuficiencia. Esto, de acuerdo con Vázquez y col. (2018), es favorable para la unidad de producción al depender menos del exterior para su funcionamiento. El G3 tiene la mayor participación femenina vinculada al trabajo reproductivo en la UPF. Según López y col. (2013), la participación de la mujer en tales actividades favorece la generación de otros ingresos y mitiga la dependencia de recursos externos al tiempo que contribuye a la seguridad alimentaria familiar $\mathrm{y}$, por ende, al sustento y desarrollo de la UPF.

\section{CONCLUSIONES}

Las Unidades de Producción Familiar (UPF) estudiadas en la Frailesca presentan un conjunto de características en su capital humano que contribuyen o afectan a corto, mediano y largo plazo la conservación de los maíces locales, como la continuidad generacional, el deterioro de los recursos naturales que sostienen el cultivo a través de la fusión de las prácticas tradicionales con las modernas y la dependencia de recursos externos. El capital social de las UPF se basa fundamentalmente en las relaciones locales y de cooperación, las institucionales son menos trascendentales. El G3 muestra un mejor equilibrio entre el capital humano y social por contar con mayor partici- 
pación familiar masculina y femenina, destacando entre los de mayor experiencia en el cul- tivo de maíces locales y tener relaciones locales y de cooperación más sólidas.

\section{REFERENCIAS}

Aguilar, J. C. E., Galdámez, G. J., Martínez, F. B., Guevara, H. F. y Vázquez, S. H. (2019). Eficiencia del policultivo maíz-frijol-calabaza bajo manejo orgánico en la Frailesca, Chiapas, México. Revista Científica Agroecosistemas. 7(3): 64-72.

Aguilar, R., Nahed, J., Parra, R., García, L. y Ferguson, B. (2012). Medios de vida y aproximación de sistemas ganaderos al estándar de producción orgánica en Villaflores, Chiapas, México. Avances en Investigación Agropecuaria. 16(3): 21-51.

Bartra, A. (2020). Repensar lo rústico. Aportes a una teoría del campesinado contemporáneo. En J. Boltvinik y S. A. Mann (Eds.), Pobreza y persistencia campesina en el siglo XXI. Teorías, debates, realidades y políticas (pp.113-133). Ciudad de México: Editores Siglo XXI.

Cadena-Iñiguez, P., Garrido, L. K. I., Rendón, M. R., Rangel, Q. J., Salinas, C. E. y Fernández, G. I. (2016). Persistencia campesina: estrategias de vida en áreas marginadas de Chiapas. Revista Mexicana de Ciencias Agrícolas. 7(4): 809-819.

Cadena-Iñiguez, P., Guevara, F., Argüello, R. A. y Rendón, R. (2018a). Proceso de comunicación, extensionismo y adopción de tecnologías. Revista Mexicana de Ciencias Agrícolas. 9(4): 851-864.

Cadena-Iñiguez, P., Rendón, R., Rodríguez V, H., Camacho V, C., Santellano E, E., Guevara H, F. y Govaerts, B. (2018b). Ensayo: Propueta metodológica-interinstitucional para un nuevo extensionismo en México. Revista Mexicana de Ciencias Agrícolas. 9(8): 17777-1785.

Cadena-Iñiguez, P., Rodríguez H, R., Morales G, M., Berdugo R, J. G., Salinas C, E., Camas G, R., ..., and Pinto R, R. (2019). Model of Management for Innovation in Small Farmers of Latin America; A Successful Proposal. International Journal of Agriculture and Natural Resources. 6(1):1-7.

Campos, S. R. A., Cruz, M. W. O. y Céspedes, O. E. (2019). Pensando en políticas públicas de sustentabilidad ambiental desde el análisis de la actividad productiva de la región Frailesca. Revista Legislativa de Estudios Sociales y de Opinión Pública. 12(26): 11-42.

Delgado, R. F., Guevara, H. F. y Acosta, R. R. (2018). Criterios campesinos para la selección de maíz (Zea maiz L.) en Villaflores y Villa Corzo, Chiapas. CienciaUAT. 13(1): 123-134.

FAO, Food and Agriculture Organization (2018). Mé- xico rural del siglo XXI. [En línea]. Disponible en: http: //www.fao.org/3/i9548es/I9548ES.pdf. Fecha de consulta: 12 de enero de 2020.

García, B. D. V., Pérez, Z. L. A., Pérez, B. J. J., Ruíz, R. O. y García, P. E. (2015). Sostenibilidad en una unidad de producción familiar en el trópico subhúmedo del estado de Veracruz. [En línea]. Disponible en: https://www. researchgate.net/publication/313473494_Sostenibilidad_ en_una_unidad_de_produccion_familiar_en_el_tropico_ subhumedo_del_estado_de_Veracruz. Fecha de consulta: 12 de enero de 2020.

Gobierno del estado de Chiapas (2014). Programa regional de desarrollo 2013-2018. Región VI Frailesca. [En línea].Disponible en: http://www.observatoriomujereschiapas.org.mx/descargas/ped-chiapas.pdf. Fecha de consulta: 12 de enero de 2020.

Gómez, W., Sánchez, E., Espinoza, A. y Herrera, F. (2013). El papel de los activos productivos en modos de vida rurales. La obtención de indicadores. Convergencia. Revista de Ciencias Sociales. 20(62): 71-105.

Guevara, F. G., Hernández, M. A., Basterrechea, J. L., Fonseca, M. A., Delgado, R. F, Ocaña, G. M. y Acosta, R. R. (2020). Riqueza de maíces locales (Zea mays L.) en la región Frailesca, Chiapas, México: un estudio etnobotánico. Revista Facultad de Agronomía, LUZ. 37: 1-25.

Guevara, F., Hernández, M. A., Basterrechea, J. L., Pinto, R., Venegas, J. A., Rodríguez, L. A. y Cadena, P. (2019). Maíces locales; una contextualización de identidad tradicional. Revista de la Facultad de Ciencias Agrarias UNCuyo. 51(1): 369-381.

Guevara, H. F., Hernández, R. M. A., Pinto, R. R., Arias, Y. I., Rodríguez, L. L. A., Medina, S. L. y Rodríguez, R. S. (2018a). Oportunidades para la innovación de sistemas tradicionales de producción agropecuaria: un análisis socioantropológico retrospectivo. Ciencia Ergo-Sum. 26(1): 1-19.

Guevara, H. F., Rodríguez, L. L. A., Díaz, J. J., Pinto, R. R., Ley-de-C. A. y Aryal, D. R. (2018b). Actores y estrategias de la innovación tecnológica en la producción de maíz en Chiapas, México. Revista Facultad de Agronomía, LUZ. 35(2): 223-247.

Hernández, M. A., Rodríguez, L., Guevara, F., Rosales, M. A., Pinto, R. y Ortiz, R. (2017). Caracterización Molecular de maíces locales de la reserva de la Biosfera, La 
Sepultura.México.AgronomíaMesoamericana.28(1):69-83.

Hernández, R. M. A., Guevara, F., Basterrechea, J. L., Coutiño, B., La-O, M. A., y Pinto, R. . (2020). Diversidad y conservación de maíces locales de la Frailesca, Chiapas, México. Revita Fitotecnia Mexicana.43(4):471-479.

Hernández, S. R., Fernández, C. C. y Baptista, L. M. P. (2014). Metodología de la investigación (Sexta edición). Ciudad de México: McGRAW-HILL/INTERAMERICANA EDITORES, S.A. DE C.V. 634 Pp.

López, G. J. L., Damián, H. M. A., Álvarez, G. F., Zuluaga, S. G. P., Parra, I. F. y Paredes, S. J. A. (2013). El traspatio de los productores de maíz: en San Nicolás de los Ranchos, Puebla-México. Ra Ximhai. 9(2): 181-198.

Maletta, H. (2011). Tendencias y perspectivas de la agricultura familiar en América Latina. Documento de trabajo No. 1. Proyecto Conocimiento y cambio en pobreza rural y desarrollo. Santiago de Chile. Rimisp. $131 \mathrm{Pp}$.

Martínez-Aguilar, F. B. (2021). La sustentabilidad del agroecosistema maíz (Zea mays L.) en la región Frailesca, Chiapas, México. Tesis de Ciencias Agropecuarias y Sustentabilidad, UNACH. [En línea]. Disponible en: http:// www.repositorio.unach.mx:8080/jspui/handle/1234 56789/3383. Fecha de consulta: 16 de noviembre de 2020.

Martínez, F. B, Guevara, F., Aguilar, C. E., Pinto, R., LaO, M. A., Rodríguez, L. A., and Aryal, D. R. (2020b). Energy and Economic Efficiency of Maize Agroecosystem under Three Management Strategies in the Frailesca, Chiapas (Mexico). Agriculture.10(81): 1-16.

Martínez, F. B., Guevara, C. E., Aguilar, L. A., Rodríguez, M. B. y Reyes, M. B. (2020a). Caracterización físico-química y biológica del suelo cultivado con maíz en sistemas convencional, agroecológico y mixto en la Frailesca, Chiapas. Terra Latinoamericana. 38(4): 871-881.

Martínez, F. B, Guevara, F., La-O, M. A., Rodríguez, L. A., Pinto, R. y Aguilar, C. E. (2020c). Caracterización de productores de maíz e indicadores de sustentabilidad en Chiapas. Revista Mexicana de Ciencias Agrícolas. 11(5): 1031-1042.

Pizaña, V. H. A., Fletes, O. H. B. y González, C. A. A. (2019). Agronegocios y campesinos maiceros en la Frailesca, en Eutopía, Revista de Desarrollo Económico Territorial 15. [En línea]. Disponible en: http://www.revistas.flacsoandes.edu.ec. Fecha de consulta: 4 de octubre de 2019.

Rubio, B. (2012). El declive de la fase agroexportadora Neoliberal en el contexto de las crisis capitalista y alimentaria. En M. Hernández y J. Meléndez (Eds.), Alimentación contemporánea: Un paradigma de crisis y respuestas alternativas (pp. 31-77). México: Editorial CLAVE.

Salcedo, S. y Guzmán, L. (2014). Agricultura familiar en
América Latina y el Caribe. Recomendaciones de politica. Santiago de Chile: Organización de la Naciones Unidas para la Alimentación y la Agricultura. 486 Pp.

Santos, V., Zúñiga, M., Rodríguez J. y Álvarez, A. (2014). Tipologías de productores agropecuarios para la orientación de políticas públicas: Aproximación a partir de un estudio de caso en la región Texcoco. Estado de México. México. Sociedades Rurales, Producción y Medio Ambiente. 14(28): 47-69.

SIAP, Servicio de Información Agroalimentaria y Pesquera (2020). Secretaría de Agricultura, Ganadería, Desarrollo Rural, Pesca y Alimentación. [En línea]. Disponible en: http://nube.siap.gob.mx/cierre_agricola/. Fecha de consulta: 18 de agosto de 2020 .

SJR, Servicio Jesuita a Refugiados (2017). Manual Medios de Vida. Bogotá. Colombia: Editorial de la Oficina Regional JSR LAC. 50 Pp.

Van-der-Ploeg, J. (2013). Diez cualidades de la agricultura familiar. LEISA. Revista de Agroecología. 29(4): 6-21.

Toledo, V. (2008). Metabolismos rurales: hacia una teoría ecológica-económica de la apropiación de la naturaleza. Revista Iberoamericana de Economía Ecológica. 7: 1-26.

Vázquez, G. L. B., Parra, V. M. R. y Gracia, M. A. (2018). Transformaciones en la agricultura de los mayas peninsulares: Un contraste de los casos de Kampocolché y Xohuayán. Mundo Agrario. 19(41): e084.

Yúñez, A., Cisneros, A. y Meza, P. (2013). Situando la agricultura familiar en México. Principales características y tipos, en Serie Documentos de Trabajo No 149. RIMISP, Santiago de Chile. [En línea]. Disponible en: http://www. portalsiget.net. Fecha de consulta: 18 de noviembre de 2018. 